\title{
Dacomitinib in lung cancer: a "lost generation" EGFR tyrosine-kinase inhibitor from a bygone era?
}

\author{
This article was published in the following Dove Press journal: \\ Drug Design, Development and Therapy \\ 15 October 2015 \\ Number of times this article has been viewed
}

\section{Sai-Hong Ignatius $\mathrm{Ou}^{\prime}$ \\ Ross A Soo ${ }^{2}$}

'Chao Family Comprehensive Cancer Center, Division of Hematology/ Oncology, Department of Medicine, University of California, Irvine School of Medicine, Orange, CA, USA; ${ }^{2}$ National University Health System and Cancer Science Institute of Singapore, Singapore
Correspondence: Sai-Hong Ignatius Ou Chao Family Comprehensive Cancer Center, Division of Hematology/ Oncology, Department of Medicine, University of California, Irvine School of Medicine, I0I City Drive South, Orange, CA 92868, USA

$\mathrm{Tel}+\mathrm{I} 7144568104$

Fax + I 7|4 4562242

Email ignatius.ou@uci.edu
Abstract: EGFR tyrosine-kinase inhibitors (TKIs) have now been firmly established as the first-line treatment for non-small-cell lung cancer (NSCLC) patients harboring activating EGFR mutations, based on seven prospective randomized Phase III trials. However, despite significantly improved overall response rate and improved median progression-free survival when compared to platinum-doublet chemotherapy, EGFR-mutant NSCLC patients treated with EGFR TKIs invariably progress due to the emergence of acquired resistances, with the gatekeeper T790M mutation accounting for up to $60 \%$ of the resistance mechanisms. Second-generation irreversible EGFR TKIs were developed in part to inhibit the T790M mutation, in addition to the common activating EGFR mutations. Dacomitinib is one such second-generation EGFR TKI designed to inhibit both the wild-type (WT) EGFR and EGFR T790M. Afatinib is another second-generation EGR TKI that has been now been approved for the first-line treatment of EGFR-mutant NSCLC patients, while dacomitinib continues to undergo clinical evaluation. We will review the clinical development of dacomitinib from Phase I to Phase III trials, including the two recently published negative large-scale randomized Phase III trials (ARCHER 1009, NCIC-BR-26). Results from another large-scale randomized trial (ARCHER 1050) comparing dacomitinib to gefitinib as first-line treatment of advanced treatment-naïve EGFR-mutant NSCLC patients will soon be available and will serve as the lynchpin trial for the potential approval of dacomitinib in NSCLC. Meanwhile, third-generation EGFR TKIs (eg, CO-1686 [rociletinib], AZ9291, HM61713, EGF816, and ASP8273) that preferentially and potently inhibit EGFR T790M but not WT EGFR are in full-scale clinical development, and some of these EGFR TKIs have received "breakthrough" designation by the US Food and Drug Administration and will likely be approved in late 2015. Given the rapid development of third-generation EGFR TKIs and the approval of gefitinib, erlotinib, and afatinib as first-line treatment of EGFR-mutant NSCLC patients, the future role of dacomitinib in the treatment of NSCLC seems to be limited.

Keywords: dacomitinib, epidermal growth factor receptor (EGFR), tyrosine-kinase inhibitor (TKI), EGFR T790M, second-generation EGFR TKI

\section{Introduction}

The discovery of activating EGFR mutations in non-small-cell lung cancer (NSCLC) in 2004 heralded the era of molecular classification of lung cancer and precision cancer medicine in lung cancer. ${ }^{1-3}$ Although it was not until 2009 when the first retrospective analysis from the seminal IPASS trial demonstrated that testing for activating $E G F R$ mutations matters in adenocarcinoma of the lung in determining whether EGFR tyrosinekinase inhibitor (TKI) or platinum-doublet chemotherapy is more efficacious. Prior to that, it is generally accepted that clinical characteristics, such as Asian ethnicity, 
adenocarcinoma, female sex, and never-smoking status, are sufficient to enrich for NSCLC patients who may benefit from EGFR TKIs, and testing for activating EGFR mutation is expensive and hence unnecessary. ${ }^{4}$ IPASS also demonstrated that EGFR TKIs should optimally be used as first-line treatment of NSCLC patients harboring activating EGFR mutations. ${ }^{4}$ In the next 3 years, seven randomized trials comparing EGFR TKIs to doublet platinum-based chemotherapy were published or presented confirming and cementing that EGFR TKIs is the standard of care for NSCLC patients harboring activating EGFR mutations..$^{5-11}$ All these trials demonstrated statistical significant improvement in overall response rate (ORR) and progression-free survival (PFS) with EGFR TKIs over doublet chemotherapy in EGFR-mutated NSCLC patients. However, the median PFS for NSCLC patients with EGFR-activating mutations is in the range of 10-14 months, as invariably almost all patients will progress on EGFR TKIs. One of the main mechanisms of acquired resistance to EGFR TKIs is the emergence of gatekeeper T790M mutation. in several large-scale $(>20$ patients) retrospective studies that investigated the acquired-resistance mechanism to EGFR TKIs in EGFR-mutated NSCLC patients, the prevalence of T790M gatekeeper alone or in conjunction with other potential resistance mechanisms was about $60 \% .^{12-15}$

\section{In vitro and nonhuman clinical pharmacology of dacomitinib}

Dacomitinib is designed and synthesized as a pan-HER inhibitor, while first-generation EGFR TKIs (gefitinib and erlotinib) effectively inhibit EGFR only. The inhibitory concentrations needed to inhibit $50 \%\left(\mathrm{IC}_{50}\right)$ against the purified kinase activities of the wild-type (WT) EGFR, ERBB2 and ERBB4 by dacomitinib is $6.0 \mathrm{nM}, 45.7 \mathrm{nM}$ and $73.7 \mathrm{nM}$ respectively. In contrast, the $\mathrm{IC}_{50}$ for gefitinib against WT EGFR, ERBB2 and ERBB4 kinase is $3.1 \mathrm{nM}, 343 \mathrm{nM}$ and $476 \mathrm{nM}$ respectively, and the $\mathrm{IC}_{50}$ for erlotinib is $0.56 \mathrm{nM}$, $512 \mathrm{nM}$, and $790 \mathrm{nM}$, respectively. ${ }^{16}$ Even as a pan-HER inhibitor, dacomitinib is still better at inhibiting EGFR than other members of the HER family, and has comparable but not superior $\mathrm{IC}_{50}$ against WT EGFR when compared to firstgeneration EGFR TKIs. In addition to inhibiting the two most common activating EGFR mutations (exon 19 deletion and L858R substitution), dacomitinib also has inhibitory activity in gefitinib-resistant cell-line models harboring EGFR exon 20 T790M resistance mutations. The in vitro $\mathrm{IC}_{50}$ of dacomitinib against the cell lines HCC827 (exon 19 del E746_A750), and H3255 (L858R substitution) is $0.002 \mathrm{M}$ and $0.0007 \mathrm{M}$, respectively, and for gefitinib, the $\mathrm{IC}_{50}$ is $0.008 \mathrm{M}$ and $0.075 \mathrm{M}$, respectively, highlighting the potency of dacomitinib against the two common EGFR mutations. Furthermore, gefitinib does not inhibit T790M whilst dacomitinib has potential inhibitory activity. ${ }^{16}$ However, it should be noted that the $\mathrm{IC}_{50}$ against the T790M for dacomitinib is in the range of hundreds of nanomolars. ${ }^{16}$ In animal studies, dacomitinib has high bioavailability $(>50 \%)$, long half-life ( $>12$ hours), and large volume of distribution $(>17 \mathrm{~L} / \mathrm{kg})$ in rats, monkeys, and dogs. ${ }^{17}$

\section{Clinical development of dacomitinib (ARCHER)}

The development of dacomitinib in NSCLC by Pfizer is under the acronym ARCHER (Advanced Research for Cancer targeted pan-HER therapy), and will be used throughout this review to list the major dacomitinib trials in NSCLC. The list of dacomitinib trials in this review is in Table 1.

\section{ARCHER I00I (US Phase I study), ARCHER 1003 (South Korea Phase I study),ARCHER 1005 (Japan Phase I study)}

Three Phase I studies of dacomitinib have been conducted in the US, Japan, and South Korea, and are listed in Table 2. ${ }^{18-20}$ The US trial had the most patients, but all three trials revealed similar side effects of dacomitinib that are also typical of EGFR TKIs (rash, stomatitis, diarrhea, paronychia, dry skin), with diarrhea, stomatitis, rash, and palmar-plantar erythrodysesthesia as the dose limiting toxicities determined from the US Phase I trial. ${ }^{18}$ All three Phase I trials arrived at the same recommended Phase II dose of $45 \mathrm{mg}$ of dacomitinib orally once daily. There did not seem to be a food effect on the pharmacokinetic level of dacomitinib. ${ }^{18}$ The half-life of dacomitinib was estimated to be 59-85 hours in the US Phase I study. ${ }^{18}$ Although not stated, the half-life of dacomitinib in Japanese patients seemed to be similar to US patients. ${ }^{19}$

There was an expanded cohort of the US Phase I trial where 57 NSCLC patients were enrolled and treated with $45 \mathrm{mg}$ once daily of dacomitinib. A total of 33 of the 57 NSCLC patients harbored EGFR mutations, including exon 19 deletion, L858R, exon 20 insertion, and exon 19 deletion/T790M. Of the 21 NSCLC patients harboring the common EGFR mutations, two patients achieved partial responses $(9.5 \%)$, eleven patients achieved stable disease (SD; 52.3\%), and eight patients had progressive disease (PD; 38.1\%) as the best response. Of the four NSCLC patients with exon 19 deletion/T790M mutation, two achieved SD and two PD as the best response. 
Table I List of dacomitinib trials in this review

\begin{tabular}{|c|c|c|c|}
\hline ARCHER & NCT number & Description & References \\
\hline \multicolumn{4}{|c|}{ Single-arm Phase I } \\
\hline 1001 & 00225121 & US Phase I trial & 18 \\
\hline 1003 & 00553254 & South Korea Phase I trial & 20 \\
\hline 1005 & 00783328 & Japan Phase I trial & 19 \\
\hline \multicolumn{4}{|c|}{ Single-arm Phase II } \\
\hline 1002 & 00548093 & US Phase II trial, KRAS WT & 21 \\
\hline 1017 & $008|844|$ & $\begin{array}{l}\text { Single-arm first-line treatment of never-smoker/light former smoker or in EGFR-mutant } \\
\text { NSCLC patients }\end{array}$ & 28 \\
\hline \multicolumn{4}{|c|}{ Randomized Phase II } \\
\hline 1028 & 00769067 & $\begin{array}{l}\text { Randomized Phase II dacomitinib versus erlotinib as second-line treatment in unselected } \\
\text { NSCLC }\end{array}$ & 26 \\
\hline \multicolumn{4}{|c|}{ Randomized Phase III } \\
\hline 1009 & 01360554 & Randomized dacomitinib versus erlotinib in second-line treatment of unselected NSCLC & 27 \\
\hline BR-26 & 01000025 & $\begin{array}{l}\text { Randomized dacomitinib versus placebo in third-line or beyond treatment of unselected } \\
\text { NSCLC }\end{array}$ & 28 \\
\hline 1050 & 01774721 & $\begin{array}{l}\text { Randomized dacomitinib versus erlotinib as first-line treatment of EGFR-mutant (exon } \\
19 \text { deletion or L858R) NSCLC }\end{array}$ & 29 \\
\hline
\end{tabular}

Abbreviations: WT, wild type; NSCLC, non-small-cell lung cancer.

Of the six NSCLC patients with exon 20 insertion, one had a partial response, two had SD, and two had $\mathrm{PD}$, with one nonevaluable. Interestingly, two NSCLC patients had HER2 amplified, and both achieved SD as the best response. In the Phase II portion of the Phase I/II South Korean trial, the estimated PFS at 4 months was $47.2 \%$ (95\% confidence interval
[CI] 31.6\%-61.3\%), with a median PFS of 15.4 weeks ( 3.6 months; $95 \%$ CI 9.7-17.6 weeks) and median overall survival (OS) of 46.3 weeks ( 10.8 months; $95 \%$ CI 32.7-not reached) for the 43 KRAS WT NSCLC patients. ${ }^{20}$ Altogether, the initial clinical activity of dacomitinib is promising, but similar to the first-generation EGFR TKIs (gefitinib, erlotinib).

Table 2 Comparison of the results of the three dacomitinib Phase I studies

\begin{tabular}{|c|c|c|c|}
\hline & $\mathbf{U S}^{18}$ & South Korea ${ }^{20}$ & Japan $^{19}$ \\
\hline $\mathrm{n}$ & 121 & 12 & 13 \\
\hline ClinicalTrials.gov NCT number & 00225121 & 00553254 & 00783328 \\
\hline ARCHER & 1001 & 1003 & 1005 \\
\hline \multirow[t]{5}{*}{ Tumor types } & Solid malignancies & KRAS WT NSCLC, prior erlotinib & Solid malignancies \\
\hline & NSCLC (45\%) & or gefitinib failure, more than one & NSCLC (69\%) \\
\hline & Colorectal (22\%) & systemic regimen & Colorectal (15\%) \\
\hline & Breast $(6 \%)$ & & Breast $(8 \%)$ \\
\hline & Ovarian (5\%) & & \\
\hline Dose range & $0.5-60 \mathrm{mg}$ & $30-45 \mathrm{mg}$ & $15-45 \mathrm{mg}$ \\
\hline DLTs & $\begin{array}{l}\text { Diarrhea, stomatitis, } \\
\text { rash, palmar-plantar } \\
\text { erythrodysesthesia }\end{array}$ & None & None \\
\hline RP2D & 45 mg orally daily & 45 mg orally daily & 45 mg orally daily \\
\hline Grade 4 toxicities ( $>5 \%)$ & None & None & None \\
\hline \multirow[t]{4}{*}{ Grade 3 toxicities ( $>5 \%)$} & Diarrhea $(9.9 \%)$ & None & Diarrhea $(28.6 \%)$ \\
\hline & Dermatitis acneiform (5.4\%) & & Decreased appetite (14.3\%) \\
\hline & & & ALT increased (14.3\%) \\
\hline & & & AST increased (14.3\%) \\
\hline \multirow[t]{5}{*}{ Most common toxicities } & Grade I diarrhea (39\%) & Grade 2 dermatitis acneiform (50\%) & Grade $\mathrm{I}-3$ rash (I00\%) \\
\hline & Grade I rash (27.0\%) & Grade I diarrhea (42\%) & Grade I-3 diarrhea (92\%) \\
\hline & Grade I dry skin (26.2\%) & Grade I paronychia (33.3\%) & Grade I-3 paronychia (69\%) \\
\hline & Grade I nausea (25.2\%) & Grade I stomatitis (33.3\%) & Grade $1-3$ dry skin (62\%) \\
\hline & & & Grade I-3 stomatitis (62\%) \\
\hline Food effect & None & Not investigated & Not investigated \\
\hline
\end{tabular}

Abbreviations: NSCLC, non-small-cell lung cancer; WT, wild type; DLTs, dose limiting toxicities; RP2D, recommended Phase II dose; n, number of patients. 


\section{Single-arm Phase II studies of dacomitinib (clinical and molecular selection)}

There are two important single-arm medium-size studies ( $<100$ patients) of dacomitinib (ARCHER 1002, ARCHER 1017) that investigated the potential roles of dacomitinib in the treatment of two molecularly defined cohorts of NSCLC patients (KRAS WT and EGFR-mutated), respectively.

\section{ARCHER 1002 (KRAS wild type) (A747 I002; NCT00548093)}

ARCHER 1002 was a single-arm study of dacomitinib in patients with NSCLC who failed erlotinib and at least one and no more than two chemotherapy regimens (neoadjuvant/ adjuvant/investigational regimens did not count). Patients had to be either KRAS WT by the Scorpion Amplified Refractory Mutation System allele-specific polymerase chainreaction assay or assumed to be KRAS WT if the tumor was shown to harbor the common activating EGFR mutations (exon 19 deletion, L858R), with ORR as the primary end point. Interestingly, the primary end point was analyzed in two different patient cohorts (adenocarcinoma versus nonadenocarcinoma). ${ }^{21}$

The ORR was $4.8 \%$ and $6.3 \%$ for adenocarcinoma and nonadenocarcinoma patients, respectively, thus not reaching the primary end point specified for adenocarcinoma patients $(>=5 \%)$ but the end point for nonadenocarcinoma patients $(>=1 \%)$. The ORR among EGFR-mutant patients was only $8 \%$, with a disease-control rate at 6 weeks of $68 \%$ (Table 3). There was no RECIST (Response Evaluation Criteria In Solid Tumors) response by dacomitinib among the six T790M EGFR-mutant patients. ${ }^{21}$ Of note, the ORR and PFS in KRAS WT NSCLC patients was similar to the results reported for erlotinib ${ }^{22}$ or gefitinib. ${ }^{23}$ Although the patients enrolled were heavily pretreated and the sample size was small, the low ORRs seen in patients with adenocarcinoma, EGFR T790M patients, and EGFR-mutant patients were disappointingly low.

\section{ARCHER 1017 (never-smokers/light former smokers or EGFR-mutant) (A747 I0I 7; NCT008I844I)}

ARCHER 1017 was a second single-arm Phase II study of dacomitinib in advanced treatment-naïve NSCLC patients with clinicopathologic characteristics enriched for activating EGFR mutations (adenocarcinoma, never-smokers defined

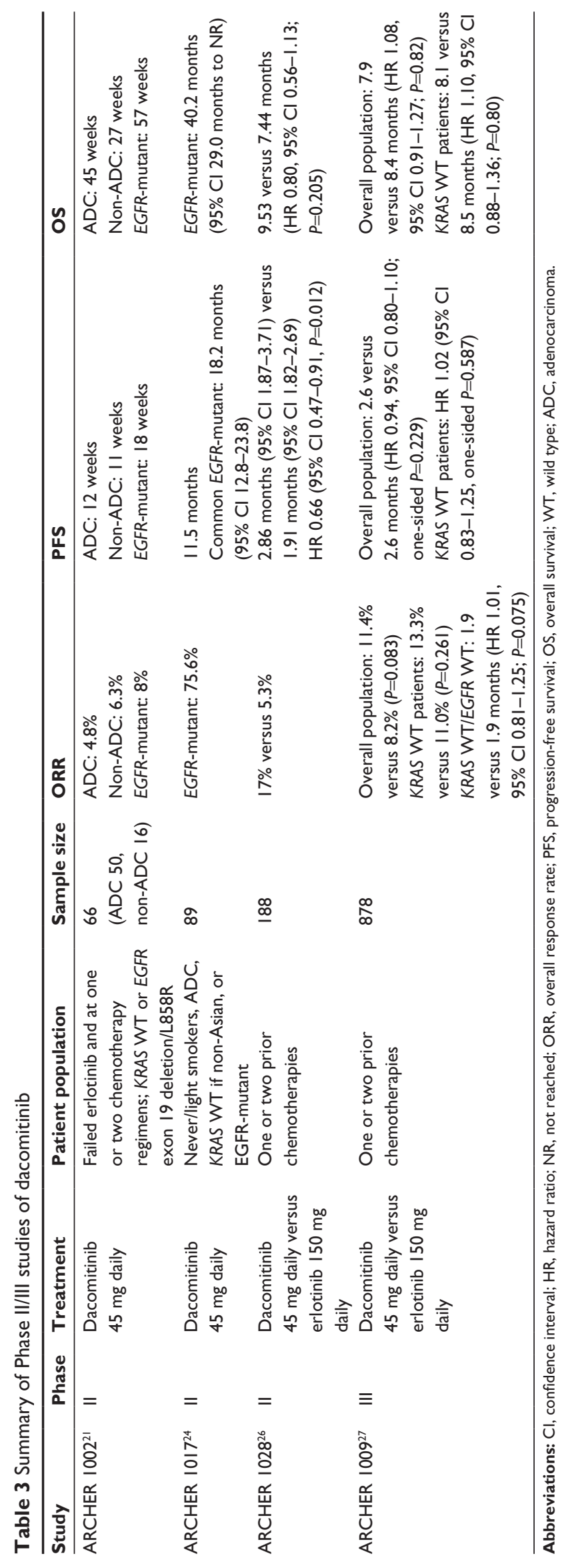


as $<100$ cigarettes lifetime or light former smokers defined as $<10$ pack-years or $\geq 15$ years since last cigarette and $K R A S$ WT if non-Asian) or EGFR-mutant NSCLC patients regardless of smoking status. ${ }^{24}$ The EGFR-mutant cohort was added to the trial after the IPASS data ${ }^{4}$ were presented indicating the activity of EGFR TKI is most efficacious in molecularly defined EGFR-mutant patients rather than using clinical characteristics to select for patients for EGFR TKIs. Patients enrolled on the basis of clinical factors were retrospectively analyzed for EGFR mutations on available tissue. The primary end point of the study was PFS at 4 months, but an independent central review of radiologic response was not conducted. A second phase of the study in patients who had HER 2 mutation or HER 2 gene amplification was begun and has not been reported.

The 4-month PFS rate was 76.8\%, and the median PFS was 11.5 months. There was also clinically meaningful improvement in quality of life in terms of dyspnea, chest pain, and cough. A total of 53 of the enrolled patients had a confirmed EGFR mutation (45 with the common EGFR mutations: exon 19 deletion [ $\mathrm{n}=25$ ] or exon 21 L858R substitution [n=20]). Importantly, the median PFS in these patients with the two common EGFR mutations was 18.2 months, and ORR was $75.6 \%$ (95\% CI 60.5-87.1). Furthermore, the estimated median OS was 40.2 months for the 45 patients with the two common EGFR mutations (Table 5). There was no significant difference between patients with exon 19 deletion and patients with L858R substitution, respectively, in terms of ORR (75\% versus $76 \%)$, median PFS (16.6 months [95\% CI 11.5-25.7] versus 18.3 months [95\% CI 9.2-24.8]), or estimated OS (40.0 months [95\% CI 23.0-not reached] versus 46.0 months [95\% CI 24.8-not reached]). The median PFS achieved in EGFR-mutant patients for dacomitinib in even this Phase II study was impressive, and similar to the median PFS achieved with afatinib in LUX-Lung-2 (15.6 months for exon 19 deletion as first-line treatment [ $\mathrm{n}=17]$ and 16.0 months for L858R as first-line treatment $[\mathrm{n}=14]$; all investigator-assessed), ${ }^{25}$ and provided expectation that the ARCHER 1050 trial (discussed later) would be positive.

Equally importantly, the ORR, median PFS, and estimated median OS for the 14 EGFR WT NSCLC patients were $7.1 \%$ (95\% CI 0.2-33.9), 2.1 months (95\% CI 2.9-7.4), and 19.7 months (95\% CI 3.5-24.3), respectively. ${ }^{24}$ Although the number of EGFR WT patients was small, these data are further indication that the clinical activity of dacomitinib is similar to the first-generation EGFR TKIs (gefitinib and erlotinib).

\section{Randomized Phase II trial of dacomitinib ARCHER 1028 (dacomitinib versus erlotinib as second-line treatment in unselected NSCLC patients) (NCT00769067)}

Dacomitinib was compared to erlotinib as second-line treatment of unselected NSCLC patients in this 1:1 randomized open-label study. Stratification factors for the randomization included race (Asian versus non-Asian), smoking status (never-smoker versus ever-smoker), and histology (adenocarcinoma versus nonadenocarcinoma). The primary end point of the trial was $\geq 45 \%$ improvement in median PFS with dacomitinib over erlotinib. ${ }^{26}$

A statistically significant improvement in median PFS was seen in patients treated with dacomitinib compared to erlotinib, with a stratified log-rank hazard ratio (HR) of $0.66(95 \%$ CI 0.47-0.91, $P=0.012$ ). The calculated stratified log-rank HR for the primary end point took into account $E G F R$-mutation status, KRAS-mutation status, and Eastern Cooperative Oncology Group performance status. The median PFS for patients with activating EGFR mutations was 7.44 months, regardless of whether they were treated with dacomitinib or erlotinib (HR 0.46, 95\% CI 0.18-1.18). Similarly, there was no difference in median PFS among KRAS-mutated patients, regardless of dacomitinib or erlotinib treatment (HR 0.99, 95\% CI 0.45-2.17). There was no difference in median PFS among two of the three stratification factors for randomization: race and smoking status. Dacomitinib resulted in significantly improved PFS among adenocarcinoma patients compared to erlotinib (HR 0.62, 95\% CI 0.41-0.94). Not unexpectedly for a randomized Phase II trial, there was no significant difference in OS between the two treatment arms (Table 3).

ORR was a statistically better among dacomitinib-treated patients $(17.0 \%)$ than erlotinib-treated patients $(5.3 \%$, $P=0.011$ ). However, treatment arms were imbalanced, with $20.2 \%$ of the dacomitinib-treated patients harboring activating EGFR mutations compared to only $11.7 \%$ of the erlotinib-treated patients. Of note, the median PFS of dacomitinib-treated patients decreased from 3.71 months for $K R A S \mathrm{WT} / E G F R$ any type-only patients to 2.21 months for both $K R A S$ WT and EGER WT patients, indicating the PFS for dacomitinib was influenced by the presence of activating $E G F R$ mutations. Given there was no difference in median PFS between dacomitinib-treated and erlotinib-treated EGFRmutant patients in this study, the best representative of WT NSCLC patients should have had the KRAS WT/EGFR WT 
phenotype. Indeed, the most significant finding of the trial was that dacomitinib treatment $(n=39)$ narrowly but statistically significantly improved median PFS over erlotinib treatment (n=51) among KRAS WT/EGFR WT patients (2.21 months versus 1.84 months, HR $0.61,95 \%$ CI $0.37-0.99 ; P=0.043) .{ }^{26}$ The results of ARCHER 1028 indicated dacomitinib can potentially confer improved PFS over erlotinib in both unselected and KRAS WT/EGFR WT NSCLC patients, and provided the rationale for launching the pivotal randomized Phase III ARCHER 1009 trial (described later).

Side effects attributable to EGFR TKIs were more prevalent among dacomitinib-treated patients than erlotinibtreated patients: diarrhea (73.1\% versus $47.8 \%)$, dermatitis acneiform $(64.5 \%$ versus $57.4 \%)$, dry skin $(23.7 \%$ versus $14.9 \%)$, and stomatitis $(29.0 \%$ versus $10.6 \%)$. Of note, grade 3 diarrhea ( $11.8 \%$ versus $4.3 \%$ ), and grade 3 dermatitis (10.8\% versus $6.4 \%$ ) acneiform were more common among dacomitinib-treated patients. These side effects were reflected in the more severe symptom scores in patient-reported outcomes. Dacomitinib-treated patients reported significantly more severe diarrhea symptoms during the first two cycles (56 days) of treatment and significantly more severe mouthsore symptoms during the first three cycles (84 days) of treatment. Furthermore, the symptom scores for diarrhea and sore mouth were always worse for dacomitinib than erlotinib throughout the duration of the trial. Paronychia, which is uncommon among first-generation EGFR TKIs, was quite common among dacomitinib-treated patients $(25.8 \%)$ when compared to erlotinib-treated patients $(8.5 \%){ }^{26}$ Therefore, in any pivotal trial involving dacomitinib, the benefit in PFS improvement has to exceed the potential downside of increased frequency and severity of side effects associated with dacomitinib treatment.

\section{Randomized Phase III trials of dacomitinib} ARCHER 1009 (dacomitinib versus erlotinib in unselected NSCLC patients [second-line and third line setting]) (NCTOI 360554)

ARCHER 1009 was a multinational, multicenter, randomized, double-blinded, Phase III study comparing the efficacy and safety of second-line treatment with dacomitinib to treatment with erlotinib in patients with advanced-stage NSCLC previously treated with at least one prior regimen. Primary end points were improvement in PFS among the whole population and among KRAS WT patients. Despite the improved PFS in
$K R A S$ WT/EGFR-any-type patients from ARCHER 1028, which may have been influenced by the higher proportion of EGFR mutations among dacomitinib-treated patients, and despite the observation that median PFS was significantly improved among KRAS WT/EGFR WT patients with dacomitinib, KRAS WT/EGFR WT patients were not chosen as the patient population where the coprimary end point would be analyzed in this pivotal Phase III trial. Stratifications were by histology (adenocarcinoma versus nonadenocarcinoma), race (non-Asian versus Asian and Indian subcontinent), Eastern Cooperative Oncology Group performance status (0-1 versus 2), and smoking status (never-smoker versus ever-smoker). KRAS and EGFR mutations were analyzed with Scorpion Amplified Refractory Mutation System polymerase chain reaction (Qiagen NV, Venlo, the Netherlands). ${ }^{27}$

Of the patients enrolled, 59.1\% harbored KRAS WT, $77.7 \%$ were EGFR WT, $68.7 \%$ had adenocarcinoma histology, and $18.3 \%$ were never-smokers. Adverse events of all grades from dacomitinib-treated patients $(94.0 \%)$ were significantly more than erlotinib-treated patients $(90.0 \%$, $P=0.0249$ ), primarily due to increased incidences of all grades of diarrhea ( $72 \%$ versus $47 \%, P<0.0001$ ), all grades of stomatitis (37\% versus $20 \%, P<0.0001)$, and all grades of paronychia $(21 \%$ versus $11 \%, P=0.0001) .{ }^{27}$ There was no difference in outcomes between the two treatment arms in the overall population and in the KRAS WT for ORR, PFS, or OS (Table 3). Furthermore KRAS WT/EGFR WT patients did not benefit more from dacomitinib either, as the median PFS was identical for both groups of patients (Table 3). ${ }^{27}$

Subgroup analysis did not reveal any clinicopathologic patient characteristics, including age, sex, ethnicity, smoking status, or histology favoring either dacomitinib or erlotinib. KRAS WT/EGFR WT groups were composed of roughly equal proportions of dacomitinib-treated (49.9\%) and erlotinib-treated $(50.3 \%)$ patients. Finally, there was no difference in the composite time to deterioration of pain, dyspnea, fatigue, or cough (HR 0.90, 95\% CI 0.76-1.07; P=0.77). ${ }^{27}$ In all, ARCHER 1009 was a pivotal negative trial, with dacomitinib not superior to erlotinib in terms of ORR, PFS, OS, or quality of life, while it had significantly more toxicity than erlotinib.

\section{NCIC-BR-26 (dacomitinib versus placebo as third-line or beyond treatment in unselected NSCLC patients) (NCTOI000025)}

In this Canadian National Cancer Institute of Cancer-sponsored randomized Phase III trial, dacomitinib was compared to 
placebo in one of the largest Phase III randomized placebocontrolled trials as third-line or beyond (after failure of chemotherapy and an EGFR TKI) treatment of advanced unselected NSCLC. OS was the primary end point of the trial, with the sample-size calculations based on 33\% improvement in OS (from 4.0 months to 5.3 months) based on $90 \%$ power and one-sided significance level of $2.5 \%$. One of the secondary end points also included OS in KRAS WT patients and in $E G F R$-mutant patients. Stratification factors included centers, performance status, smoking status, ethnicity, best response to prior EGFR TKIs, and weight loss. ${ }^{28}$

A total of 720 patients were enrolled and randomized in a 2:1 ratio to dacomitinib and placebo, respectively; $26 \%$ of the patients had had one prior line of chemotherapy (plus one prior EGFR TKI), indicating that dacomitinib/placebo was third-line treatment for these patients. Furthermore, 59\% of the patients had had two lines of chemotherapy, indicating that this was fourth-line treatment for these patients, because they also had to have failed one EGFR TKI. Another $12 \%$ of the patients had had three or more lines of chemotherapy, indicating these were really heavily pretreated (dacomitinib/placebo as fifth line of treatment) patients. The majority ( $75.3 \%)$ of the patients had performance status $0-1,48.5 \%$ were $E G F R$ WT, $25.3 \%$ were $E G F R$-mutant, and $10.8 \%$ were $K R A S$ mutations. In addition, $28.9 \%$ of the patients had $\mathrm{PD}$ as the best response to prior EGFR TKIs, while $42.5 \%$ had SD and only $12.9 \%$ had complete/partial response as best response to prior EGFR TKIs. In about $15.7 \%$ of patients, the best response to prior EGFR TKIs was unknown. ${ }^{28}$ The results of the published Phase II/III trials are summarized in Table 3.

NCIC-BR-26 failed to achieve its primary end point, as there was no statistically significant improvement in median OS among dacomitinib-treated patients (6.83 months, $95 \%$
CI 6.08-7.49) compared to placebo-treated patients $(6.31$ months, 95\% CI 5.32-7.52; HR 1.00; 95\% CI 0.83-1.21; $P=0.9873)$. Analysis of predefined secondary end points showed no difference in median OS in EGFR-mutant patients between dacomitinib treatment (7.23 months, $95 \%$ CI 6.08-8.61) and placebo treatment (7.52 months, $95 \%$ CI 4.99-9.49) (HR 0.98; 95\% CI 0.67-1.44; $P=0.461)$ or improvement in median OS in KRAS WT patients between dacomitinib-treated (7.0 months, 95\% CI 6.01-8.21) and erlotinib-treated patients (5.19 months, 95\% CI 4.53-7.00) (HR 0.79, 95\% CI 0.61-1.30; $P=0.043$ ). There was no improvement in OS among EGFR-mutant patients (HR $0.981,95 \%$ CI $0.669-1.439 ; P=0.9218)$ either. However, $K R A S$-mutant patients who received dacomitinib had significantly worse OS (5.8 months versus 8.3 months, HR $2.10,95 \%$ CI $1.05-4.22 ; P=0.984)$. Although not the primary end point of the trial, median PFS was significantly improved with the use of dacomitinib over placebo (2.7 months versus 1.4 months, HR 0.66 , 95\% CI $0.55-0.79 ; P<0.0001$ ), and ORR was significantly better than placebo ( $7 \%$ versus $1 \%$, $P=0.001) .{ }^{28}$ The median OS and PFS according to the $E G F R$ and KRAS genotype were listed in Table 4. The median OS and PFS according to best response to prior EGFR TKIs are listed in Table 5. This subset analysis indicated that dacomitinib was more active in patients who did not progress from prior EGFR TKIs.

Patients who received dacomitinib had significantly longer time to deterioration of cough (12 months versus 4.6 months, $P<0.0001$ ), dyspnea (5.6 months versus 4.6 months, $P=0.049$ ) and pain (3.0 months versus 1.9 months, $P=0.041$ ). As expected, more side effects were seen in dacomitinib treated patients, including diarrhea ( $78 \%$ versus $15 \%$ ), acneiform rash ( $58 \%$ versus $8 \%$ ), oral mucositis ( $41 \%$ versus

Table 4 Summary of median overall survival (OS) and progression-free survival (PFS) between dacomitinib and placebo in NCIC-BR-26

\begin{tabular}{|c|c|c|c|c|c|c|c|}
\hline & \multicolumn{2}{|c|}{ Dacomitinib } & \multicolumn{2}{|l|}{ Placebo } & \multirow[t]{2}{*}{ HR } & \multirow[t]{2}{*}{$95 \% \mathrm{Cl}$} & \multirow[t]{2}{*}{ P-value } \\
\hline & Median & $95 \% \mathrm{Cl}$ & Median & $95 \% \mathrm{Cl}$ & & & \\
\hline \multicolumn{8}{|c|}{ Median OS (months) } \\
\hline All & 6.83 & $6.08-7.49$ & 6.31 & $5.32-7.52$ & 1.00 & $0.83-\mid .21$ & 0.506 \\
\hline KRAS WT & 7.00 & $6.01-8.21$ & 5.19 & $4.53-7.00$ & 0.79 & $0.61-1.03$ & 0.043 \\
\hline EGFR-mutant & 7.23 & $6.08-8.61$ & 7.52 & 4.99-9.49 & 0.98 & $0.67-1.44$ & 0.461 \\
\hline EGFR WT & 6.93 & $5.82-8.08$ & 5.55 & $4.60-7.20$ & 0.93 & $0.7|-| .21$ & 0.283 \\
\hline KRAS-mutant & 5.82 & $4.11-7.23$ & 8.28 & $4.27-14.90$ & 2.10 & $1.05-4.22$ & 0.984 \\
\hline \multicolumn{8}{|c|}{ Median PFS (months) } \\
\hline All & 2.66 & $1.91-3.33$ & $\mathrm{I} .38$ & $0.99-1.74$ & 0.66 & $0.55-0.79$ & $<0.0001$ \\
\hline KRAS WT & 3.06 & $1.91-3.55$ & 1.05 & $0.9-1.7 \mid$ & 0.58 & $0.46-0.73$ & \\
\hline EGFR-mutant & 3.52 & $2.53-3.68$ & 0.95 & $0.89-1.64$ & 0.48 & $0.35-0.66$ & \\
\hline EGFR WT & 1.91 & I.77-2.79 & 1.63 & $0.99-\mid .81$ & 0.75 & $0.59-0.95$ & \\
\hline KRAS-mutant & 1.61 & $0.92-1.87$ & 1.86 & $0.95-2.33$ & 1.34 & $0.78-2.29$ & \\
\hline
\end{tabular}

Note: Data from Ellis et al. ${ }^{28}$

Abbreviations: $\mathrm{Cl}$, confidence interval; $\mathrm{HR}$, hazard ratio. 
Table 5 Summary of median overall survival (OS) and progression-free survival (PFS) between dacomitinib and placebo according to previous response to EGFR TKIs in NCIC-BR-26

\begin{tabular}{|c|c|c|c|c|c|c|c|}
\hline \multirow[t]{2}{*}{ Best response to EGFR TKIs } & \multicolumn{2}{|c|}{ Dacomitinib } & \multicolumn{2}{|l|}{ Placebo } & \multirow[t]{2}{*}{ HR } & \multirow[t]{2}{*}{$95 \% \mathrm{Cl}$} & \multirow[t]{2}{*}{ Interaction $P$-value } \\
\hline & Median & $95 \% \mathrm{Cl}$ & Median & $95 \% \mathrm{Cl}$ & & & \\
\hline \multicolumn{8}{|l|}{ Median OS (months) } \\
\hline Progressive disease & 5.49 & $4.73-6.28$ & 7.52 & $5.16-9.13$ & 1.36 & $1.00-1.87$ & \\
\hline Others & 7.56 & $6.77-8.28$ & 6.01 & $5.13-7.33$ & 0.79 & $0.65-0.97$ & 0.003 \\
\hline \multicolumn{8}{|l|}{ Median PFS (months) } \\
\hline Progressive disease & 1.71 & $1.12-1.84$ & 1.74 & $0.92-2.04$ & 1.05 & $0.78-|.4|$ & \\
\hline Others & 3.52 & $2.83-3.61$ & 1.12 & $0.95-|.7|$ & 0.56 & $0.46-0.68$ & 0.001 \\
\hline
\end{tabular}

Note: "Others" included complete/partial response, stable disease, and unknown. Data from Ellis et al. ${ }^{28}$

Abbreviations: $\mathrm{Cl}$, confidence interval; TKIs, tyrosine-kinase inhibitors; $\mathrm{HR}$, hazard ratio.

$3 \%$ ), dry skin $(30 \%$ versus $7 \%$ ), paronychia (28\% versus $0 \%$ ), fatigue $(22 \%$ versus $12 \%)$, nausea (20\% versus $9 \%$ ), and vomiting ( $20 \%$ versus $2 \%$ ), although whether these differences were significant was not reported. ${ }^{28}$

\section{ARCHER 1050 (dacomitinib versus gefitinib as first-line treatment of advanced EGFR-mutant NSCLC patients) (NCTOI77472I)}

ARCHER 1050 is an ongoing multicenter, randomized, openlabel, Phase III study comparing dacomitinib to gefitinib in treatment-naïve advanced EGFR-mutant NSCLC patients harboring the common-activation EGFR mutations (exon 19 deletions and L858R substitution). The presence of T790M is allowed as long as one of the two common EGFR mutations is also present. The primary end point is PFS, and randomization is one to one. This trial is being conducted only in Asia (People's Republic of China, Japan, South Korea, Hong Kong) and Europe (Spain, Italy, Poland), and not in North America. The goal of the trial is to show $>50 \%$ improvement in median PFS in dacomitinib over gefitinib, with an estimated median PFS of erlotinib of 9.5 months and projected median PFS of dacomitinib to be at least 14.3 months. Stratifications are by type of EGFR mutation and ethnicity. ${ }^{29}$ The trial schema are represented in Figure 1.

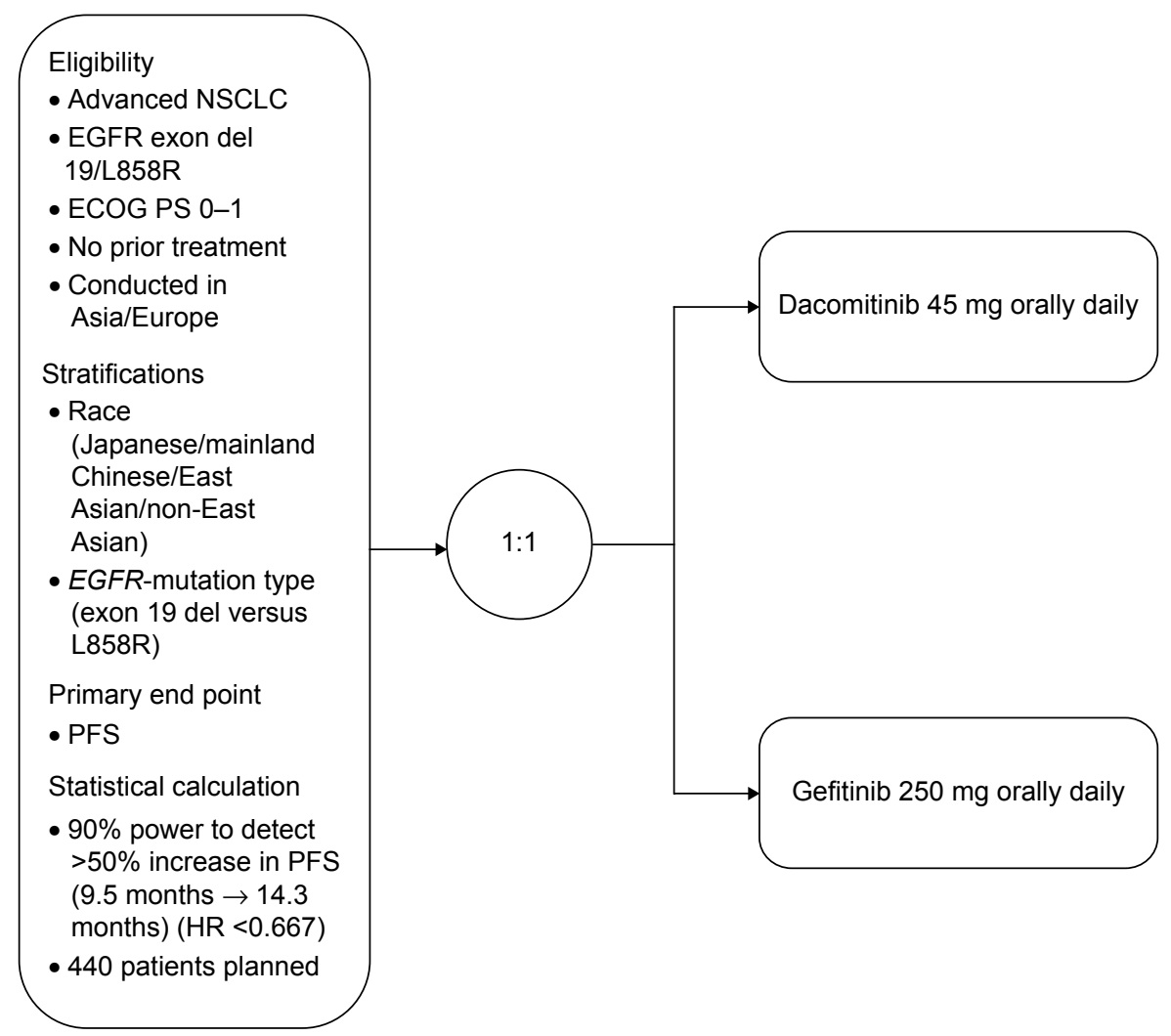

Figure I Study design for ARCHER 1050.

Abbreviations: NSCLC, non-small-cell lung cancer; ECOG PS, Eastern Cooperative Oncology Group performance status; PFS, progression-free survival; HR, hazard ratio. 


\section{Concluding perspectives \\ Does the use of EGFR TKIs in unselected or wild-type EGFR NSCLC represent a bygone era?}

Erlotinib received full approval in the US as second- or thirdline treatment of unselected NSCLC based on the improved OS over placebo from the NCIC-BR21 trial in 2004. 22,30 Subsequently, erlotinib was approved for "switch maintenance" in unselected NSCLC patients who had benefited from first-line platinum-based chemotherapy based on statistically significant improved albeit 1-month prolongation of OS (SATURN) in 2010. ${ }^{31,32}$ More recently, erlotinib was approved as first-line treatment of advanced $E G F R$-mutant NSCLC based on significantly improved PFS from the EURTAC trial in $2013 .^{8,33}$ In the intervening decade since the first approval of erlotinib in the US, we have gained a much better appreciation of the clinical efficacy of EGFR TKIs. Two randomized trials (TaiLOR, DELTA) comparing single-agent chemotherapy to erlotinib as second-line treatment of unselected NSCLC reported chemotherapy had statistically significant improved PFS compared with erlotinib. ${ }^{34,35}$ Against the backdrop of this evolving standard of care with EGFR TKIs, the failure of ARCHER 1009 and NCIC BR-26 to achieve their primary end points has dealt a significant setback to the near-term approval and potential use of dacomitinib in NSCLC. The results of ARCHER 1009 indicated there is no difference in terms of efficacy between dacomitinib and erlotinib as second-line treatment of unselected NSCLC regardless of histology, but more toxicities with dacomitinib. However, ARCHER 1009 was not powered to be a noninferiority trial, and thus dacomitinib cannot claim to be clinically equivalent to erlotinib. In a recently presented trial designed similarly to ARCHER 1009, except it was conducted only in squamous cell carcinoma of the lung as second-line treatment (LUX-Lung-8), afatinib when compared to erlotinib demonstrated significant improvement in disease-control rate (46\% [afatinib] versus $37 \%$ [erlotinib], $P=0.0203$ ) and duration of response (9.2 months [afatinib] versus 3.8 months [erlotinib]). Additionally, afatinib achieved its primary end point of improved median PFS in LUX-Lung-8, where the median PFS of afatinib-treated patients was 2.4 months compared to 1.9 months in erlotinib-treated patients (HR $0.822,95 \%$ CI $0.676-0.998$; log-rank $P=0.998$ ) as secondline treatment in squamous cell carcinoma of the lung. ${ }^{36}$ The absolute improvement in PFS with afatinib over erlotinib was only half a month, and was also associated with increased toxicities. Recently LUX-Lung- 8 demonstrated a statistically improvement in OS for afatinib over erlotinib (median 7.9 [afatinib] vs 6.8 mos [erlotinib]; HR [95\% CI] 0.81 [0.69-0.95]; $P=0.008) .{ }^{37}$ Whether afatinib will gain additional regulatory approval as second-line treatment of unselected squamous cell carcinoma of the lung based on achieving the primary end point statistically, improved PFS and significant improved OS remained to be determined given the new agents approved for second line $\mathrm{SqCC}$ of the lung; similarly with the failure of dacomitinib to improve upon OS over placebo in BR-26 after failure of first-generation EGFR TKI, which was similar to the results of the LUXLung-1 trial, where there was improvement in PFS with afatinib over placebo but no difference in $\mathrm{OS},{ }^{38}$ though subset analysis revealed there was OS benefit in patients whose best response to first-generation EGFR TKIs was not PD. In summary, dacomitinib failed to replace first-generation EGFR TKIs as second-line treatment of unselected NSCLC and failed to improve OS survival after disease progression in first-generation EGFR TKIs in unselected NSCLC.

Recently, two large-scale meta-analyses demonstrated for WT NSCLC patients chemotherapy is superior to EGFR TKIs in terms of PFS benefit. ${ }^{39,40}$ The ever-increasing clinical data argue against using EGFR TKIs in EGFR WT patients as second-line treatment or even using EGFR TKIs as "switch maintenance". As such, the use of EGFR TKIs in unselected or EGFR WT NSCLC patients may reflect the practice of a bygone era, when the knowledge on the optimal clinical use of EGFR TKIs was incomplete. In addition, based on two pivotal studies, alternative second-line treatment options now include the addition of an antiangiogenic agent combined with docetaxel in the second-line setting. Firstly, the addition of ramucirumab, a monoclonal antibody against the VEGFR2 inhibitor, to single-agent docetaxel as second-line treatment (REVEL) resulted in significant improvement in OS over docetaxel alone in unselected NSCLC patients regardless of histologies, ${ }^{41}$ and this combination was approved for use after platinum-based chemotherapy by the US Food and Drug Administration (FDA) in December 2014 regardless of histology. In a second Phase III study of nintedanib, an angiokinase inhibitor, combined with docetaxel versus docetaxel alone in patients with advanced-stage NSCLC (LUME-Lung 1), an improvement in OS was seen in patients with adenocarcinoma treated with combination therapy, ${ }^{42}$ leading to the approval of this combination by the European Medicines Agency in the second-line setting. ${ }^{42}$ Furthermore recently the antiPD-L1 monoclonal antibody, nivolumab, has demonstrated improvement in OS over docetaxel in both squamous and non-squamous NSCLC. ${ }^{43,44}$ 


\section{Role of dacomitinib in the first-line treatment of advanced EGFR-mutant NSCLC patients}

Given the promising published Phase II data on dacomitinib as first-line treatment of EGFR-mutant NSCLC, one would expect dacomitinib to confer superior PFS over gefitinib in $E G F R$-mutant patients, but whether this will translate to a $50 \%$ improvement of PFS (HR <0.67) in ARCHER 1050 remains to be determined. However gefitinib and erlotinib are entrenched as first-line treatment of EGFR-mutant patients with a better side-effect profile and likely represent lower cost to the payers/patients, especially when some of key patent indicators for gefitinib will expire in many countries by the end of 2017. Furthermore, afatinib was approved as first-line treatment of advanced EGFR-mutant NSCLC in 2013 based on the LUX-Lung-3 trial. ${ }^{9}$ While afatinib may have more side effects than the first-generation EGFR TKIs, recent data indicated that afatinib resulted in statistically significant improved OS in EGFR-mutant patients harboring exon 19 deletion compared to cisplatin/pemetrexed chemotherapy, indicating a potential advantage of second-generation EGFR TKIs over first-generation EGFR TKIs. ${ }^{45}$ Therefore, clinicians will not only be looking at 50\% improvement in PFS over gefitinib in ARCHER 1050 but also potential statistical improvement in OS among patients harboring EGFR exon 19 deletions, although the comparison will be made against gefitinib instead of chemotherapy, which may be less effective. The ability to produce a statistically significant improvement in OS in EGFR exon 19-deletion patients compared to a firstgeneration EGFR TKI will be an important clinical determination factor in gaining widespread adoption for dacomitinib. Finally, given ARCHER 1050 is conducted exclusively outside North America, whether the US FDA will approve dacomitinib based on a pivotal trial conducted exclusively outside the US/North America remains unknown, although this approval it is not an absolute requirement for a pivotal registration to enroll US/North America patients.

Regardless, scientifically one of the important correlative sciences that needs to emerge from ARCHER 1050 is to identify the resistance mechanisms to dacomitinib as first-line treatment of EGFR-mutant NSCLC patients. It has been reported that T790M-resistance mutation can occur after progression on afatinib as first-line treatment of EGFR-mutant patients. ${ }^{46}$ It remains to be seen whether resistance developed against dacomitinib will involve T790M mutation. However, if precedence holds, dacomitinib is unlikely to overcome the emergence of T790M, given there were no responders in ARCHER 1002. The $\mathrm{IC}_{50}$ of dacomitinib against $\mathrm{T} 790 \mathrm{M}$ is in the $100-500 \mathrm{nM}$ range. The rapid clinical development of at least five thirdgeneration EGFR TKIs (CO-1686, AZD9121, HM61713, EFG816, and ASP8273) that target specifically the T790M mutation but not WT EGFR needs to be taken into consideration as well. The $\mathrm{IC}_{50}$ against $\mathrm{T} 790 \mathrm{M}$ among these thirdgeneration EGFR TKIs are in the single-digit nanomolar range (Table 6) ${ }^{47-49}$ Furthermore, these compounds have

Table 6 List of $I C_{50}$ values (nano-molar) among first-generation (gefitinib, erlotinib), second-generation (afatinib, dacomitinib), and third-generation (AZD929I, CO-I686, HM78I-36B, ASP8273) EGFR TKIs against various EGFR genotypes

\begin{tabular}{|c|c|c|c|c|c|c|c|c|c|}
\hline & \multicolumn{2}{|c|}{$\begin{array}{l}\text { First-generation EGFR } \\
\text { TKIs }\end{array}$} & \multicolumn{2}{|c|}{$\begin{array}{l}\text { Second-generation EGFR } \\
\text { TKIs }\end{array}$} & \multicolumn{4}{|c|}{ Third-generation EGFR TKIs } & \multirow[t]{2}{*}{ References } \\
\hline & Gefitinib & Erlotinib & Afatinib & Dacomitinib & AZD929 I & CO-1686 & HM6I7I3 & ASP8273 & \\
\hline \multicolumn{10}{|c|}{ EGFR WT cell line } \\
\hline Calu3 & 1,933 & 4,101 & 71 & 65 & 650 & NA & NA & NA & 44 \\
\hline $\mathrm{NCl}-\mathrm{H} 2073$ & 200 & 692 & 30 & 54 & 461 & NA & NA & NA & 44 \\
\hline $\mathrm{NCl}-\mathrm{HI} 666$ & 46 & 48 & 2.8 & 8.1 & 110 & 770 & NA & 230 & 45 \\
\hline H358 & NA & 449 & 31 & NA & NA & NA & 2,225 & NA & 46 \\
\hline \multicolumn{10}{|c|}{ EGFR exon 19-deletion cell line } \\
\hline PC-9 & 23 & 28 & 0.8 & 0.4 & 8 & NA & NA & NA & 44 \\
\hline PC-9 & 34 & 46 & 3.0 & 17 & 62 & 350 & NA & 19 & 45 \\
\hline $\mathrm{NCl}-\mathrm{HI} 650$ & 5,100 & 9,900 & 77 & 76 & 340 & 840 & NA & 70 & 45 \\
\hline HCC827 & 7.5 & 9.8 & 0.76 & 0.55 & 4.3 & 45 & NA & 9.9 & 45 \\
\hline $\mathrm{HCC} 827$ & NA & 3.2 & 1.8 & NA & NA & NA & 9.2 & NA & 46 \\
\hline \multicolumn{10}{|c|}{ EGFR dell9/T790M cell line } \\
\hline PC-9 VanR & 4,232 & 5,778 & 679 & 531 & 40 & NA & NA & NA & 44 \\
\hline PC-9ER & 2,000 & 1,500 & 23 & 27 & 14 & 100 & NA & 14 & 45 \\
\hline \multicolumn{10}{|c|}{ EGFR L858R/T790M cell line } \\
\hline NCl-HI975 & 6,962 & 6,165 & 483 & 335 & 11 & NA & NA & NA & 44 \\
\hline $\mathrm{NCl}-\mathrm{HI} 975$ & 9,700 & 10,000 & 230 & 110 & 28 & 140 & 26 & NA & 45 \\
\hline NCl-HI975 & NA & 2,253 & 53 & NA & NA & NA & 10 & NA & 46 \\
\hline
\end{tabular}

Abbreviations: $\mathrm{IC}_{50}$, half-maximal inhibitory concentration; TKIs, tyrosine-kinase inhibitors; NA, not available. 
demonstrated significant clinical activity against T790M without the usual toxicities from inhibiting WT EGFR, such as rash or diarrhea. ${ }^{49-51}$

\section{Conclusion}

Dacomitinib is a second-generation EGF TKI designed to be a better WT EGFR TKI and also a better EGFR TKI against the two common $E G F R$ mutations and potentially the T790M gatekeeper mutation. However, dacomitinib failed to show better clinical efficacy over first-generation EGFR TKIs and with more toxicities in unselected NSCLC patients. The failure of dacomitinib to be approved anywhere in the world to date represents partially the failure to anticipate the rapid changing standard of care involving the clinical use of EGFR TKIs. The first-generation and second-generation EGFR TKIs are almost exclusively used as first-line treatment of EGFR-mutant NSCLC patients. Additionally, afatinib has demonstrated significantly improved OS as first-line treatment of EGFR-mutant NSCLC. Even if the ARCHER 1050 trial demonstrates significantly meaningful improved in PFS over firstgeneration EGFR TKIs by dacomitinib and additionally improved OS over first-generation EGFR TKIs among EGFR exon 19 patients, it is still unlikely for dacomitinib to find a major role in the treatment of EGFR-mutant NSCLC patients when it is approved, considering the cost and toxicities. Furthermore, at least two third-generation EGFR TKIs designed to overcome T790M mutation should be approved in the US by the end of 2015/early 2016, likely even ahead of the approval of dacomitinib with much less toxicity. The negative results from the ARCHER 1009 and NCIC-BR-26 trials has delayed and may have diminished the eventual approval of an important pan-HER inhibitor.

The era of enrolling molecularly unselected lung cancer patients into clinical trials involving targeted agents is coming to an end, and even if there is planned retrospective analysis of the genomic profile of the tumor, it is likely that several third-generation EGFR TKIs will be approved before the outcome of ARCHER 1050 is known. By then, it may have turned out that using a clinical development strategy unknowingly from a bygone era, dacomitinib may be a "lost generation" EGFR TKI, where it is has been bypassed from first-generation to third-generation EGFR TKIs by many oncologists.

\section{Disclosure}

The authors report no conflicts of interest in this work.

\section{References}

1. Lynch TJ, Bell DW, Sordella R, et al. Activating mutations in the epidermal growth factor receptor underlying responsiveness of non-smallcell lung cancer to gefitinib. N Engl J Med. 2004;350:2129-2139.

2. Paez JG, Jänne PA, Lee JC, et al. EGFR mutations in lung cancer: correlation with clinical response to gefitinib therapy. Science. 2004; 304:1497-1500.

3. Pao W, Miller V, Zakowski M, et al. EGF receptor gene mutations are common in lung cancers from "never smokers" and are associated with sensitivity of tumors to gefitinib and erlotinib. Proc Natl Acad Sci USA. 2004;101:13306-13311.

4. Mok TS, Wu YL, Thongprasert S, et al. Gefitinib or carboplatinpaclitaxel in pulmonary adenocarcinoma. $N$ Engl J Med. 2009;361: 947-957.

5. Mitsudomi T, Morita S, Yatabe Y, et al. Gefitinib versus cisplatin plus docetaxel in patients with non-small-cell lung cancer harbouring mutations of the epidermal growth factor receptor (WJTOG3405): an open label, randomised phase 3 trial. Lancet Oncol. 2010;11:121-128.

6. Maemondo M, Inoue A, Kobayashi K, et al. Gefitinib or chemotherapy for non-small-cell lung cancer with mutated EGFR. NEngl J Med. 2010; 362:2380-2388.

7. Zhou C, Wu YL, Chen G, et al. Erlotinib versus chemotherapy as first-line treatment for patients with advanced EGFR mutation-positive non-smallcell lung cancer (OPTIMAL, CTONG-0802): a multicentre, open-label, randomised, phase 3 study. Lancet Oncol. 2011;12:735-742.

8. Rosell R, Carcereny E, Gervais R, et al. Erlotinib versus standard chemotherapy as first-line treatment for European patients with advanced EGFR mutation-positive non-small-cell lung cancer (EURTAC): a multicentre, open-label, randomised phase 3 trial. Lancet Oncol. 2012; 13:239-246.

9. Sequist LV, Yang JC, Yamamoto N, et al. Phase III study of afatinib or cisplatin plus pemetrexed in patients with metastatic lung adenocarcinoma with EGFR mutations. J Clin Oncol. 2013;31: 3327-3334.

10. Wu YL, Zhou C, Hu CP, et al. Afatinib versus cisplatin plus gemcitabine for first-line treatment of Asian patients with advanced non-small-cell lung cancer harbouring EGFR mutations (LUX-Lung 6): an open-label, randomised phase 3 trial. Lancet Oncol. 2014;15:213-222.

11. Wu YL, Liam CK, Zhou C, et al. First-line erlotinib versus cisplatin/ gemcitabine (GP) in patients with advanced EGFR mutation-positive non-small-cell lung cancer (NSCLC): interim analysis from the phase 3, open-label, ENSURE study. J Thorac Oncol. 2013;8 Suppl 2:S603.

12. Sequist LV, Waltman BA, Dias-Santagata D, et al. Genotypic and histological evolution of lung cancers acquiring resistance to EGFR inhibitors. Sci Transl Med. 2011;3:75ra26.

13. Yano S, Yamada T, Takeuchi S, et al. Hepatocyte growth factor expression in EGFR mutant lung cancer with intrinsic and acquired resistance to tyrosine kinase inhibitors in a Japanese cohort. J Thorac Oncol. 2011; 6:2011-2017.

14. Yu HA, Arcila ME, Rekhtman N, et al. Analysis of tumor specimens at the time of acquired resistance to EGFR-TKI therapy in 155 patients with EGFR-mutant lung cancers. Clin Cancer Res. 2013;19:2240-2247.

15. Ji W, Choi CM, Rho JK, et al. Mechanisms of acquired resistance to EGFR-tyrosine kinase inhibitor in Korean patients with lung cancer. BMC Cancer. 2013;13:606.

16. Engelman JA, Zejnullahu K, Gale CM, et al. PF00299804, an irreversible pan-ERBB inhibitor, is effective in lung cancer models with EGFR and ERBB2 mutations that are resistant to gefitinib. Cancer Res. 2007; 67:11924-11932.

17. Gonzales AJ, Hook KE, Althaus IW, et al. Antitumor activity and pharmacokinetic properties of PF-00299804, a second-generation irreversible pan-erbB receptor tyrosine kinase inhibitor. Mol Cancer Ther. 2008; 7:1880-1889.

18. Jänne PA, Boss DS, Camidge DR, et al. Phase I dose-escalation study of the pan-HER inhibitor, PF299804, in patients with advanced malignant solid tumors. Clin Cancer Res. 2011;17:1131-1139. 
19. Takahashi T, Boku N, Murakami H, et al. Phase I and pharmacokinetic study of dacomitinib (PF-00299804), an oral irreversible, small molecule inhibitor of human epidermal growth factor receptor-1, -2 , and -4 tyrosine kinases, in Japanese patients with advanced solid tumors. Invest New Drugs. 2012;30:2352-2363.

20. Park K, Cho BC, Kim DW, et al. Safety and efficacy of dacomitinib in Korean patients with KRAS wild-type advanced non-small-cell lung cancer refractory to chemotherapy and erlotinib or gefitinib: a phase I/II trial. J Thorac Oncol. 2014;9:1523-1531.

21. Reckamp KL, Giaccone G, Camidge DR, et al. A phase 2 trial of dacomitinib (PF-00299804), an oral, irreversible pan-HER (human epidermal growth factor receptor) inhibitor, in patients with advanced non-small cell lung cancer after failure of prior chemotherapy and erlotinib. Cancer. 2014;120:1145-1154.

22. Shepherd FA, Rodrigues Pereira J, Ciuleanu T, et al. Erlotinib in previously treated non-small-cell lung cancer. $N$ Engl J Med. 2005;353: $123-132$.

23. Thatcher N, Chang A, Parikh P, et al. Gefitinib plus best supportive care in previously treated patients with refractory advanced non-small-cell lung cancer: results from a randomised, placebo-controlled, multicentre study (Iressa Survival Evaluation in Lung Cancer). Lancet. 2005;366: $1527-1537$.

24. Jänne PA, Ou SH, Kim DW, et al. Dacomitinib as first-line treatment in patients with clinically or molecularly selected advanced non-smallcell lung cancer: a multicentre, open-label, phase 2 trial. Lancet Oncol. 2014;15:1433-1441.

25. Yang JC, Shih JY, Su WC, et al. Afatinib for patients with lung adenocarcinoma and epidermal growth factor receptor mutations (LUX-Lung 2): a phase 2 trial. Lancet Oncol. 2012;13:539-548.

26. Ramalingam SS, Blackhall F, Krzakowski M, et al. Randomized phase II study of dacomitinib (PF-00299804), an irreversible pan-human epidermal growth factor receptor inhibitor, versus erlotinib in patients with advanced non-small-cell lung cancer. J Clin Oncol. 2012;30: 3337-3344.

27. Ramalingam SS, Jänne PA, Mok TS, et al. Dacomitinib versus erlotinib in patients with advanced-stage, previously treated non-small-cell lung cancer (ARCHER 1009): a randomised, double-blind, phase 3 trial. Lancet Oncol. 2014;15:1369-1378.

28. Ellis PM, Shepherd FA, Millward M, et al. Dacomitinib compared with placebo in pretreated patients with advanced or metastatic non-smallcell lung cancer (NCIC CTG BR.26): a double-blind, randomised, phase 3 trial. Lancet Oncol. 2014;15:1379-1388.

29. Mok T, Nakagawa K, Rosell R, et al. Phase III randomized, open label study (ARCHER 1050) of first-line dacomitinib (D) versus gefitinib (G) for advanced (adv) non-small cell lung cancer (NSCLC) in patients (pts) with epidermal growth factor receptor (EGFR) activating mutation(s). J Clin Oncol. 2013;31 Suppl:TPS8123.

30. Johnson JR, Cohen M, Sridhara R, et al. Approval summary for erlotinib for treatment of patients with locally advanced or metastatic non-small cell lung cancer after failure of at least one prior chemotherapy regimen. Clin Cancer Res. 2005;11:6414-6421.

31. Cohen MH, Johnson JR, Chattopadhyay S, et al. Approval summary: erlotinib maintenance therapy of advanced/metastatic non-small cell lung cancer (NSCLC). Oncologist. 2010;15:1344-1351.

32. Cappuzzo F, Ciuleanu T, Stelmakh L, et al. Erlotinib as maintenance treatment in advanced non-small-cell lung cancer: a multicentre, randomised, placebo-controlled phase 3 study. Lancet Oncol. 2010;11: $521-529$.

33. Khozin S, Blumenthal GM, Jiang X, et al. U.S. Food and Drug Administration approval summary: erlotinib for the first-line treatment of metastatic non-small cell lung cancer with epidermal growth factor receptor exon 19 deletions or exon 21 (L858R) substitution mutations. Oncologist. 2014;19:774-749.

34. Garassino MC, Martelli O, Broggini M, et al. Erlotinib versus docetaxel as second-line treatment of patients with advanced non-small-cell lung cancer and wild-type EGFR tumours (TAILOR): a randomised controlled trial. Lancet Oncol. 2013;14:981-988.
35. Kawaguchi T, Ando M, Asami K, et al. Randomized phase III trial of erlotinib versus docetaxel as second- or third-line therapy in patients with advanced non-small-cell lung cancer: Docetaxel and Erlotinib Lung Cancer Trial (DELTA). J Clin Oncol. 2014;32: 1902-1908.

36. Goss G, Felip E, Cobo M, et al. A randomized, open-label, phase III trial of afatinib (A) vs erlotinib (E) as second-line treatment of patients (pts) with advanced squamous cell carcinoma (SCC) of the lung following first-line platinum-based chemotherapy: LUX-Lung 8 (LL8). Ann Oncol. 2014;25 Suppl 4:iv426.

37. Soria JC, Felip E, Cobo M, et al. Afatinib (A) vs erlotinib (E) as secondline therapy of patients (pts) with advanced squamous cell carcinoma (SCC) of the lung following platinum-based chemotherapy: Overall survival (OS) analysis from the global phase III trial LUX-Lung 8 (LL8). J Clin Oncol. 2015;33:(suppl; abstr 8002).

38. Miller VA, Hirsh V, Cadranel J, et al. Afatinib versus placebo for patients with advanced, metastatic non-small-cell lung cancer after failure of erlotinib, gefitinib, or both, and one or two lines of chemotherapy (LUX-Lung 1): a phase 2B/3 randomised trial. Lancet Oncol. 2012;13: $528-538$.

39. Lee JK, Hahn S, Kim DW, et al. Epidermal growth factor receptor tyrosine kinase inhibitors vs conventional chemotherapy in non-small cell lung cancer harboring wild-type epidermal growth factor receptor: a meta-analysis. JAMA. 2014;311:1430-1437.

40. Vale CL, Burdett S, Fisher DJ, et al. Should tyrosine kinase inhibitors be considered for advanced non-small-cell lung cancer patients with wild type EGFR? Two systematic reviews and meta-analyses of randomized trials. Clin Lung Cancer. 2015;16:173-182.e4.

41. Garon EB, Ciuleanu TE, Arrieta O, et al. Ramucirumab plus docetaxel versus placebo plus docetaxel for second-line treatment of stage IV non-small-cell lung cancer after disease progression on platinum-based therapy (REVEL): a multicentre, double-blind, randomised phase 3 trial. Lancet. 2014;384:665-673.

42. Reck M, Kaiser R, Mellemgaard A, et al. Docetaxel plus nintedanib versus docetaxel plus placebo in patients with previously treated non-small-cell lung cancer (LUME-Lung 1): a phase 3, double-blind, randomised controlled trial. Lancet Oncol. 2014;15:143-155.

43. Brahmer J, Reckamp KL, Baas P, et al. Nivolumab versus Docetaxel in Advanced Squamous-Cell Non-Small-Cell Lung Cancer. $N$ Engl $J$ Med. 2015.

44. Paz-Ares L, Horn L, Borghaei H, et al. Phase III, Randomized Trial (CheckMate 057) of Nivolumab (NIVO) Versus Docetaxel (DOC) in Advanced Non-Squamous (non-SQ) Cell Non-Small Cell Lung Cancer (NSCLC). J Clin Oncol. 2015;33:(suppl; abstr LBA109).

45. Yang CHJ, Wu YL, Schuler MH, et al. Afatinib versus cisplatin-based chemotherapy for EGFR mutation-positive lung adenocarcinoma (LUXLung 3 and LUX-Lung 6): analysis of overall survival data from two randomised, phase 3 trials. Lancet Oncol. 2015;16:141-151.

46. Kim Y, Ko J, Cui Z, et al. The EGFR T790M mutation in acquired resistance to an irreversible second-generation EGFR inhibitor. Mol Cancer Ther. 2012;11:784-791.

47. Cross DA, Ashton SE, Ghiorghiu S, et al. AZD9291, an irreversible EGFR TKI, overcomes T790M-mediated resistance to EGFR inhibitors in lung cancer. Cancer Discov. 2014;4:1046-1061.

48. Sakagami H, Konagai S, Yamamoto H, et al. ASP8273, a novel mutantselective irreversible EGFR inhibitor, inhibits growth of non-small cell lung cancer (NSCLC) cells with EGFR activating and T790M resistance mutations. Cancer Res. 2014;74:1728.

49. Kim DW, Lee DH, Kang JH, et al. Clinical activity and safety of HM61713, an EGFR-mutant selective inhibitor, in advanced non-small cell lung cancer (NSCLC) patients (pts) with EGFR mutations who had received EGFR tyrosine kinase inhibitors (TKIs). J Clin Oncol. 2014; 32 Suppl 5:8011.

50. Jänne PA, Yang JC, Kim DW, et al. AZD9291 in EGFR inhibitor-resistant non-small-cell lung cancer. N Engl J Med. 2015;372:1689-1699.

51. Sequist LV, Soria JC, Goldman JW, et al. Rociletinib in EGFR-mutated non-small-cell lung cancer. $N$ Engl J Med. 2015;372:1700-1709. 


\section{Publish your work in this journal}

Drug Design, Development and Therapy is an international, peerreviewed open-access journal that spans the spectrum of drug design and development through to clinical applications. Clinical outcomes, patient safety, and programs for the development and effective, safe, and sustained use of medicines are a feature of the journal, which has also been accepted for indexing on PubMed Central. The manuscript management system is completely online and includes a very quick and fair peer-review system, which is all easy to use. Visit http://www.dovepress.com/testimonials.php to read real quotes from published authors.

Submit your manuscript here: http://www.dovepress.com/drug-design-development-and-therapy-journal 